\title{
Welcome to a New Stroke Journal
}

On behalf of the Korean Stroke Society, I am pleased to inform you that we are releasing the first issue of 'JOURNAL OF STROKE (JOS)'. In the strict sense, this is not a 'new' journal; this is a re-birth of the previous Korean Journal of Stroke into a better quality, and into an English written journal.

With strong emergence of academic drive in Asia, and worldwide interest in papers in this region, there arises a need to publish papers in English. Moreover, although Asians are suffering from unacceptable stroke burden, information in this region such as epidemiology, stroke characteristics, practice pattern and related social-economical problems, has been underrepresented and need to come out more actively in the literature.

The journal, nevertheless, is not specially focused on Asian issues, but is aiming at publishing research papers from all over the world. Although there already are several high-quality scientific journals dedicated to stroke research, given the importance of cerebrovascular diseases worldwide, we still need additional venue for publication.

The JOS is interested in every field of clinical stroke, i.e., epidemiology, risk factors, pathogenic mechanisms, diagnosis and treatment. Basic researches will also be considered if the results are closely related to clinical practice. We are now publishing JOS three times a year, but will increase the number of publications afterwards. We will try to make JOS a high standard, international journal as early as possible.

In this first issue, you can find an interesting stroke statistics in Korea, and nice reviews on Japanese epidemiology and stroke biomarkers. I hope you enjoy reading, and don't hesitate to submit your valuable works to JOS.

Finally, I would like to express my sincere thanks to the associate and assistant editors, editorial board members, and the executive committee members of the Korean Stroke Society for their tremendous help in bringing out JOS to light.

Jong S. Kim

Editor-in-chief, Journal of Stroke

Department of Neurology, Asan Medical Center, University of Ulsan College of Medicine, 88 Olympic-ro 43-gil, Songpa-gu,

Seoul 138-736, Korea

Tel: +82-2-3010-3442, Fax: +82-2- 474-4691

E-mail: jongskim@amc.seoul.kr 